\title{
ARTICLE
}

\section{Evaluation of Delivery Form of Eicosapentaenoic and Docosahexaenoic Acids During Quality Control of Fish Oil Supplements}

\author{
Tatiane Lima Amorim ${ }^{1,2}$ iD Miguel Angel de la Fuente ${ }^{2}$ Marcone Augusto Leal de Oliveira* iD \\ Pilar Gómez-Cortés ${ }^{2}$ iD \\ ${ }^{1}$ Departamento de Química, Universidade Federal de Juiz de Fora, José Lourenço Kelmer s/n, 36036-900, Juiz \\ de Fora, MG, Brazil \\ ${ }^{2}$ Instituto de Investigación en Ciencias de la Alimentación, Universidad Autónoma de Madrid, Nicolás Cabrera, 9 , \\ 28049, Madrid, Spain
}

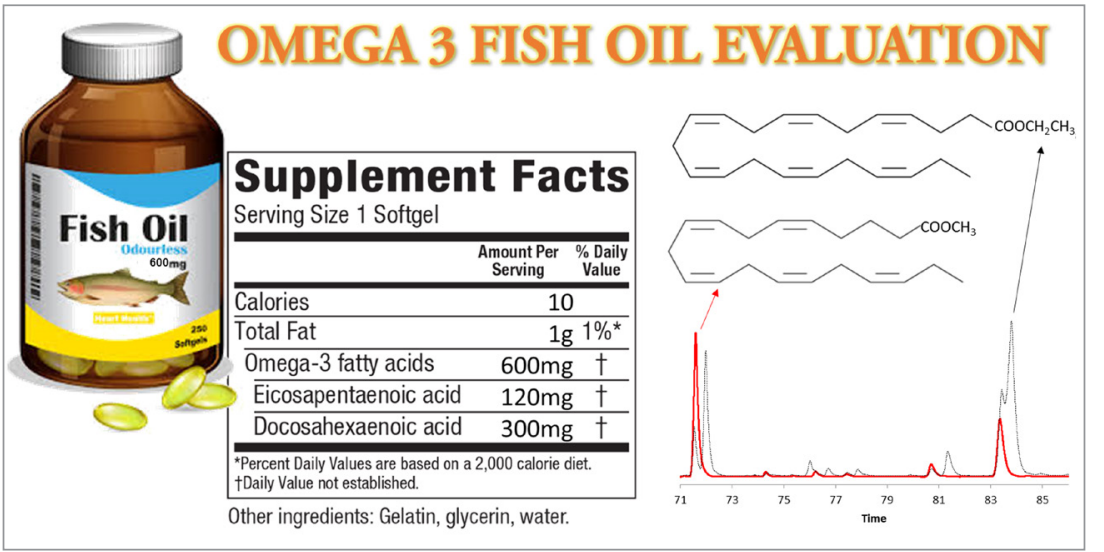

Fish oils (FO) omega-3 supplements containing eicosapentaenoic (EPA) and docosahexaenoic (DHA) acids are extensively consumed due to their beneficial health effects. Fatty acids (FA) are mainly available as triacylglycerols (TAG) and ethyl esters (EE) in FO supplements, and EE is known to be less bioavailable. Then, the evaluation of FA in FO should comprise not only omega-3 content but also the delivered form. This research aimed to approach in detail a

set of chromatographic analytical methods employing TLC, GC-FID, and GC-MS for FO quality control, considering both FA contents and form. TAG and EE FA were differentiated by GC-FID, due to the difference in the retention times of compounds, and also by GC-MS, as a result of the different $\mathrm{m} / \mathrm{z}$ spectra observed. TLC also distinguished both FA forms, but a mixture of TAG and EE in FO concentrates was not observed, as evidenced by GC techniques. Ten FO supplements available in the Brazilian market were analyzed, and the FA profiles of natural and concentrated FO were compared. EPA + DHA label claim compliance was also accessed. Their contents varied from 78 to $113 \%$ of labeled content, and only one supplement had FA in EE form. Brazilian FO supplements analyzed were a source of EPA and DHA and most products (except one sample) were accurately labeled according to current Brazilian regulation, which permits a variation in $20 \%$ of the declared content. Furthermore, the methodologies approached can be used in future researches considering FO analyses and bioavailability studies approaching the different FA forms.

Cite: Amorim, T. L.; de la Fuente, M. A.; de Oliveira, M. A.; Gómez-Cortés, P. Evaluation of Delivery Form of Eicosapentaenoic and Docosahexaenoic Acids During Quality Control of Fish Oil Supplements. Braz. J. Anal. Chem., 2020, 7 (28) pp 20-30. doi: http://dx.doi.org/10.30744/brjac.2179-3425.AR-19-2020 
Keywords: thin-layer chromatography; gas chromatography; ethyl esters fatty acids, triacylglycerols; natural fish oil; concentrated fish oil.

\section{INTRODUCTION}

Innumerable health effects of omega-3 fatty acids (FA) consumption have been largely recognized [1], especially related to the ingestion of the polyunsaturated fatty acids (PUFA) eicosapentaenoic acid (EPA) and docosahexaenoic acid (DHA), available in marine species. In the last decade, the omega-3 supplement market has grown exponentially since consumers want to reach the minimum daily recommendation of $250 \mathrm{mg}$ of EPA and DHA to obtain beneficial health-effects. Fish oil (FO) is a valuable source of EPA and DHA, being commonly commercialized in its natural or concentrated form [2]. Natural FO usually contains EPA and DHA as triacylglycerols (TAG) at different concentrations depending on the fish species, which usually is not superior to $30 \%$ of total fat. Concentrated FO are mostly available as ethyl esters (EE) since TAG resynthesis after EE distillation is costly and frequently bypassed by manufacturers [3].

The functionality of EPA and DHA as TAG or other chemical forms in FO supplements has been subject to considerable debate. In the end, a better bioavailability of the TAG form has been reported when compared to EE [4]. Currently, the delivery form of dietary FO supplements, whether TAG, EE, or other, is not legally required on the packaging in Brazil and it should be revised since consumers should be aware of which form they are choosing to understand the wide range of prices of these supplements and their different absorption forms within the body.

Moreover, inaccurate listing of EPA and DHA contents in the labels is also a core quality control issue. Recent studies worldwide evidenced that FO omega-3 supplements did not fully meet labeled EPA and DHA levels, which seriously infringe on the rights and interests of the consumers [5-7]. Regarding supplements available in the Brazilian market, Galuch et al. [8] reported that among the fifteen FO supplements analyzed (sampled in Paraná State), eleven were in the TAG form and two in the EE form. Besides, two brands were discovered with the addition of large amounts of soybean oil, which leads the final consumer to ingest low-cost oil supplements believing that they are consuming adequate doses of EPA and DHA. Overall, these findings warn the real need to intensify the inspection of dietary FO supplements worldwide. Omega-3 supplements have become one of the most popular dietary products due to the low fish intake in many populations, but these products should be more strictly controlled to ensure both quality and label declaration accuracy.

Within this context, this work aimed to apply a set of chromatographic methods described in the literature by thin-layer chromatography (TLC), gas chromatography with flame ionization detector (GC-FID) and mass spectrometry (GC-MS) for the separation of omega-3 FA in the form of TAG and EE in FO supplements. The GC method was proposed by Gallardo et al. (2014) [9] and was applied to evaluate the effects of olive and FO on the lipid composition of ewe milk, and subsequently in the muscle and subcutaneous adipose tissues of lambs suckling such milk. However, only TAG were determined. On the other hand, the TLC method was described by Srigley and Rader (2018) [10] for the identification of EE in krill oil. In the present study, ten Brazilian FO were analyzed to show the applicability of the developed methods for TAG and EE omega-3 separation, in FO supplements. The FA profiles of natural and concentrated FO were compared and discussed. Besides, EPA + DHA label claim compliance was also evaluated, which would be of great interest in a globalized market. Since the FA delivered form in supplements impacts the bioavailability of omega-3 FA in the body, this topic needs to be further addressed in the literature, and we discussed the chromatographic methods approached in detail, also showing identified chromatograms and mass spectra, which could serve for new researchers in this field. The described methodologies can be used for evaluating FO supplements quality, considering both FA composition and form. Moreover, these methods could also be investigated in future works for the determination of TAG and EE EPA and DHA in bioavailability studies. 


\section{MATERIALS AND METHODS \\ Samples}

Ten bestsellers dietary FO supplements commercialized in capsules were acquired from local markets (Juiz de Fora, Minas Gerais, Brazil, October 2018). Nine of the products were marketed for the general population (Samples 1-9), and one product was marketed toward pregnant women (Sample 10). All products declared contents of both DHA and EPA; however, no information about the delivery form of FA was provided in the labels.

\section{Reagents and analytical standards}

All reagents used for the analysis were of analytical grade. Chloroform, diethyl ether, ethanol, glacial acetic acid, methanol, hexane, potassium hydroxide $(\mathrm{KOH})$ and sodium bisulfate $\left(\mathrm{NaHSO}_{4}\right)$ were purchased from Panreac Química S.A. (Madrid, Spain). Sigma-Aldrich (St. Louis, MO, USA) provided 2',7'-dichlorofluorescein. FA methyl esters (ME) standards were acquired from Nu-Chek Prep Inc. (Elysian, MN, USA).

\section{Thin-layer chromatography}

Samples were analyzed by TLC, according to Srigley \& Orr-Tokle (2018) procedure [10]. Commercial FO samples were solubilized in chloroform $\left(25 \mathrm{mg} \mathrm{mL}^{-1}\right)$ and spotted on a silica gel $\mathrm{G}$ plate $20 \times 20 \mathrm{~cm}$ from Merck (Darmstadt, Germany). A mixture of hexane/diethyl ether/glacial acetic acid in the proportion of 100:20:2 (v/v/v) was used as the mobile phase. After developing, plates were sprayed with a $0.1 \%$ ethanolic 2',7'-dichlorofluorescein solution, and bands were visualized under ultraviolet light. TAG and EE were identified based on the calculated retention factors.

\section{Sample preparation for gas chromatography}

TAG were converted into FAME by base-catalyzed methylation before GC analysis. Omega-3 marine oils were withdrawn from the capsules with a glass syringe, and $25 \mathrm{mg}$ of fat were weighed in a microtube, where $200 \mu \mathrm{L}$ of hexane were added. After agitation, $50 \mu \mathrm{L}$ of a methanolic $\mathrm{KOH}$ solution $\left(2 \mathrm{~mol} \mathrm{~L}^{-1}\right)$ were added, and the microtube was vortexed for $1 \mathrm{~min}$. After $5 \mathrm{~min}$ of resting, $125 \mathrm{mg}$ of $\mathrm{NaHSO}_{4}$ were added, and the microtube was centrifuged for $5 \mathrm{~min}\left(10000 \mathrm{rpm}\right.$ at $\left.0^{\circ} \mathrm{C}\right)$. Finally, the solution was transferred to a GC vial and diluted in a proportion of 1:2 with hexane.

\section{Instrumentation for gas chromatography}

An Agilent 6890N GC instrument (Palo Alto, CA, USA) equipped with FID detector, autosampler, and a CP-SIL 88 capillary column $(100 \mathrm{~m}, 0.25 \mathrm{~mm} \times 0.2 \mu \mathrm{m}$, Varian, Middelburg, The Netherlands) was used to quantify FA in FO samples. The temperature program was: the initial temperature was $45^{\circ} \mathrm{C}$; after $4 \mathrm{~min}$, the temperature was raised at $13{ }^{\circ} \mathrm{C} \mathrm{min}-1$ to $165{ }^{\circ} \mathrm{C}$ and hold for $35 \mathrm{~min}$, then increased to $215^{\circ} \mathrm{C}$ at $4{ }^{\circ} \mathrm{C} \min ^{-1}$ and kept for $30 \mathrm{~min}$ [9]. An injection volume of $1.0 \mu \mathrm{L}$ and a split ratio of 1:100 were employed. FID detector and injector temperature were fixed at $250{ }^{\circ} \mathrm{C}$. Identification of EPA and DHA ME were performed in GC-FID by comparison with the retention times of FAME individual standards. Other FAME were identified by comparison with the retention times of FAME mixtures (GLC 80, 403, 409, 411, 603, 642, 643) from Nu-Chek Prep Inc. A GC-MS (electron impact ionization source and single quadrupole mass analyzer, Agilent 6890A GC, MS 5973N; Palo Alto, CA, USA) equipped with the same CP-SIL 88 capillary column was used to corroborate the identification of FAME performed by GC-FID and identify EPA and DHA EE. The filament trap current was $400 \mu \mathrm{A}$ at $70 \mathrm{eV}$; ion source and interface temperatures were 150 , and $230{ }^{\circ} \mathrm{C}$, respectively, and the mass range was $30-400 \mathrm{~m} / \mathrm{z}$. Chromatographic conditions were similar to those described by GC-FID but with a split ratio of 1:20. Wiley 275 and NIST 05 libraries were used to confirm mass spectra, which were also compared with those from The LIPID MAPS ${ }^{\circledR}$ Lipidomics Gateway. The general FA composition of commercial FO was quantified by area normalization. In addition, calibration curves for EPA and DHA were carried out by ordinary least squares regression. 


\section{RESULTS AND DISCUSSION \\ Fatty acid composition and form of natural and concentrated fish oil supplements}

Figure 1 shows the different profiles of FA obtained by GC-FID for a natural FO supplement (Figure 1-A, Sample 1) and a concentrated FO supplement (Figure 1-B, Sample 10). It can be observed that the natural FO presented a large number of major peaks besides EPA and DHA, with a high amount of saturated fatty acids (SFA), such as $\mathrm{C} 14: 0$ and C16:0, monounsaturated fatty acids (MUFA), such as C16:1 and C18:1, and some characteristics PUFA, such as C16:2, C16:3, C16:4, C18:4, C21:5, and C22:5 also reported by other researchers [11,12]. Several isomers were also separated in this natural FO (C16:1, $\mathrm{C} 18: 1, \mathrm{C} 18: 2, \mathrm{C} 18: 3,20: 4$, and 22:5). The isomeric separation was possible due to the employment of a $100 \mathrm{~m}$ cyanopropyl phase capillary column, which permits to obtain detailed information on positional and geometrical isomers of FO [13]. In the natural FO, all FA were originally present as TAG (Figure 1-A). This is the molecular form primarily present in fats and oils from both animal and plant species. In the chromatogram, all TAG are observed as MEs, since a methylation reaction was employed to convert TAG into this more volatile form.

In Figures 1-B and $-\mathrm{C}$, it can be observed that the concentrated supplement presented EPA and DHA EE as major peaks. However, a small amount of TAG can be noticed because the industrial process of molecular distillation is not $100 \%$ efficient. EE are mostly found as major FA in concentrated FO since they are formed by the esterification of TAG with ethanol for the FA distillation. As the molecular process of TAG resynthesis is highly costly, it is generally bypassed by manufacturers [3], which leads to concentrate supplements mainly composed by EE FA in the market.

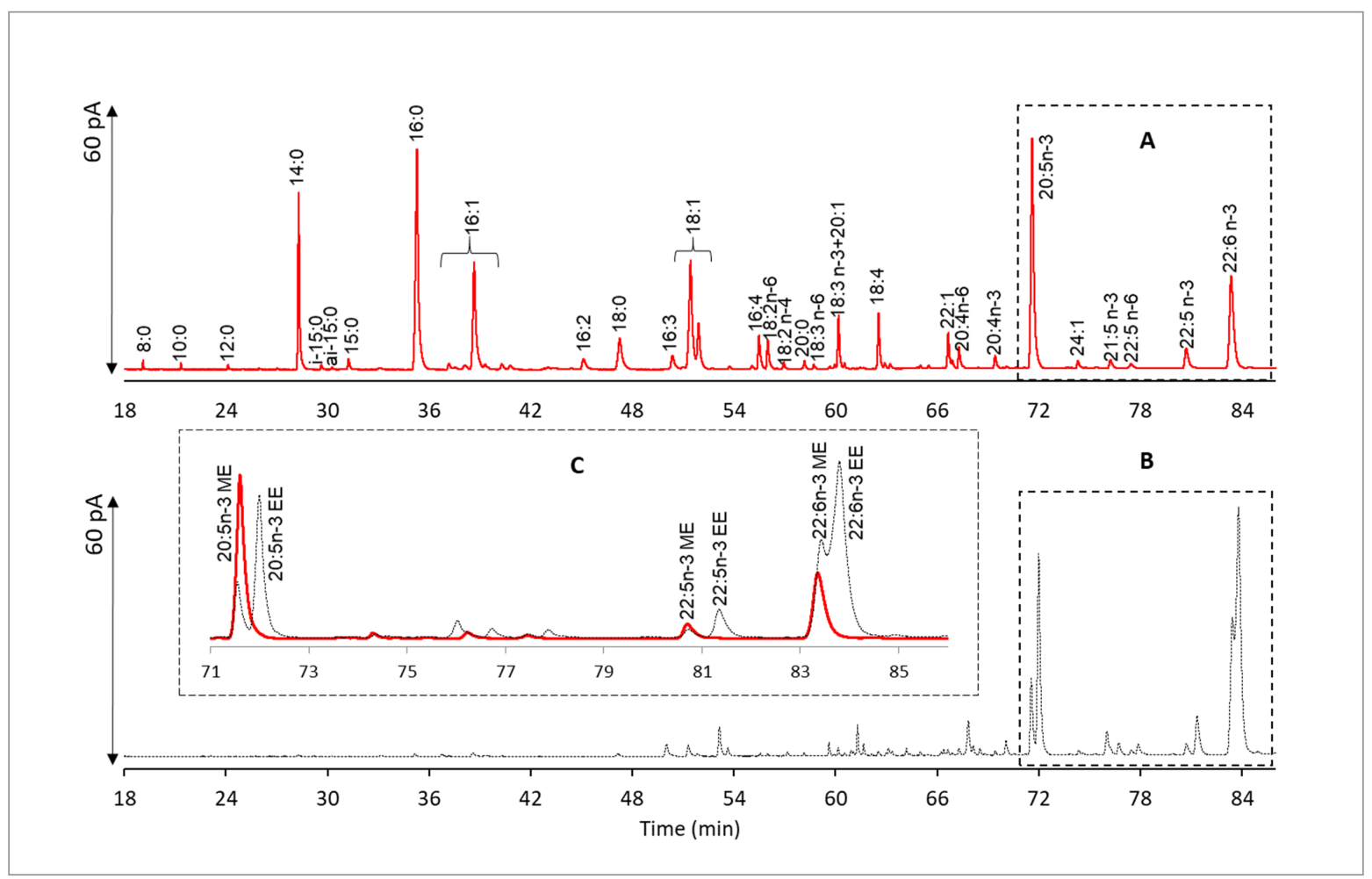

Figure 1. Chromatograms of natural fish oil ( $A$, fatty acids originally as triacylglycerols, solid red line) and concentrated fish oil (B, major fatty acids as ethyl esters (EE), dotted black line). Both samples are superimposed and amplified $(C)$ to show the elution order of methyl esters (ME) and EE omega-3 moieties. In $A$, all peaks are in the ME form. In $B$, major fatty acids are in EE form, although small amounts of ME can be observed due to the incomplete conversion of TAG to EE during supplements concentration. Triacylglycerols were analyzed as fatty acids ME after analytes derivatization. 
Table I shows the FA composition of the ten FO supplements analyzed in this study, reporting EPA and DHA contents, total SFA, including branched-chain SFA, total MUFA, and PUFA. Samples 1 to 9 presented a very similar FA profile: SFA varied from 28.4 to $30.8 \%$, MUFA from 24.2 to $28.2 \%$, and PUFA from 41.1 to $46.1 \%$. Considering EPA and DHA levels, always below $\sim 30 \%$ of total fat, it can be inferred that these supplements were natural FO encapsulated without further concentration processes (samples contained from 16.2 to $19.7 \%$ of EPA and from 9.4 to $12.4 \%$ of DHA). Besides, these FO presented FA in TAG form. Sample 10 was a concentrated omega-3 supplement marketed for pregnant women, in which EPA and DHA contents accounted for $66.7 \%$ of total fat (22.6\% EPA and $44.1 \%$ DHA). In comparison with the other samples, it presented a very different FA profile, with low SFA (only 1.9\%) and MUFA (only 3.2\%) levels. The total PUFA content was $77.8 \%$. The GC chromatogram of this concentrated sample showed that omega-3 FA were mainly in the EE form (Figure 1), accounting for $73.2 \%$ of EPA and $73.0 \%$ of DHA as EE moieties.

Table I. Fatty acid composition of commercial fish oils omega-3 dietary supplements analyzed by gas chromatography (data presented as $\%$ of total fatty acids)

\begin{tabular}{cccccc}
\hline Sample & $\boldsymbol{\Sigma S F A}^{\mathbf{a}}$ & $\boldsymbol{\Sigma}^{\mathrm{N}}$ MUFA $^{\mathbf{b}}$ & $\boldsymbol{\Sigma P U F A}^{\mathbf{c}}$ & EPA $^{\mathbf{d}}$ & DHA $^{\mathbf{d}}$ \\
\hline 1 & 29.1 & 28.0 & 41.6 & 18.3 & 10.1 \\
2 & 30.0 & 27.0 & 42.0 & 16.4 & 10.2 \\
3 & 30.3 & 26.2 & 42.4 & 16.3 & 9.7 \\
4 & 28.4 & 24.2 & 46.1 & 17.4 & 11.6 \\
5 & 28.8 & 24.2 & 45.7 & 19.7 & 12.4 \\
6 & 28.8 & 27.3 & 42.8 & 16.2 & 9.4 \\
7 & 30.8 & 27.0 & 41.1 & 17.9 & 10.0 \\
8 & 28.9 & 26.4 & 43.3 & 17.0 & 10.1 \\
9 & 28.7 & 28.2 & 42.0 & 18.0 & 10.6 \\
10 & 1.9 & 3.2 & 77.8 & 22.6 & $44.1^{\dagger}$ \\
\hline
\end{tabular}

SFA: saturated fatty acids; MUFA: monounsaturated fatty acids; PUFA: polyunsaturated fatty acids; EPA: eicosapentaenoic acid; DHA: docosahexaenoic acid.

a $\Sigma$ SFA was calculated by area normalization as the sum of 8:0, 10:0, 12:0, iso 13:0, anteiso 13:0, 13:0, iso 14:0, $14: 0$, iso 15:0, anteiso 15:0, 15:0, iso 16:0, 16:0, iso 17:0, 17:0, iso 18:0, 18:0, 19:0, 20:0, 21:0 and 22:0.

${ }^{ } \Sigma$ MUFA was calculated by area normalization as the sum of $14: 1,16: 1,17: 1,18: 1,19: 1,20: 1,22: 1$ and $24: 1$.

¿ $\Sigma$ PUFA calculated by area normalization as the sum of 16:2, 16:3, 16:4, 18:2, 18:3, 18:4, 20:2, 20:3, 20:4, 20:5, 22:2, 22:4, 22:5 and 22:6.

${ }^{d}$ calculated by external calibration. ${ }^{e} 16.4 \%$ was in the form of ethyl esters and $6.1 \%$ in the form of triacylglycerols, representing $73.2 \%$ of total EPA as ethyl esters; ${ }^{5} 32.2 \%$ was in the form of ethyl esters and $11.9 \%$ in the form of triacylglycerols, representing $73.0 \%$ of total DHA as ethyl esters.

\section{Evaluation of Brazilian fish oil supplements label accuracy}

Table II presents the comparison between the determined and labeled EPA and DHA contents in all samples analyzed considering the daily serving size recommended for each supplement. The natural FO supplements recommended three capsules as daily serving size, equivalent to $3000 \mathrm{mg}$, while the concentrated supplement intake recommendation was just one capsule, equivalent to $600 \mathrm{mg}$. When comparing labeled and analytically determined EPA and DHA contents, only sample 5 presented at least $100 \%$ of DHA claimed content and samples 1, 5, 9, and 10 at least $100 \%$ of EPA claimed content (Figure 2). 
However, when considering a tolerance of $20 \%$ in the reported nutritional value, as permitted by current Brazilian legislation [14], almost all samples were rightly labeled (except sample 6 which presented DHA $<80 \%$ of labeled content). EPA content varied from $90 \%$ to $113 \%$, considering the declared contents, and DHA concentration varied from $78 \%$ to $103 \%$. Figure 2 shows the percentage of label declaration achieved for each sample analyzed.

Table II. Label declaration and determined eicosapentaenoic (EPA) and docosahexaenoic (DHA) acid contents in the Brazilian omega-3 fish oil dietary supplements analyzed

\begin{tabular}{cccccc}
\hline & \multicolumn{2}{c}{ Labeled content } & \multicolumn{2}{c}{ Determined content } \\
\hline Sample & $\begin{array}{c}\text { Daily serving } \\
\text { size }(\mathbf{m g})\end{array}$ & $\begin{array}{c}\text { EPA } \\
\left(\mathbf{m g ~ s e r v i n g}^{-1}\right)\end{array}$ & $\begin{array}{c}\text { DHA } \\
\left(\text { mg serving }^{-1}\right)\end{array}$ & $\begin{array}{c}\text { EPA } \\
\left(\text { mg serving }^{-1}\right)\end{array}$ & $\begin{array}{c}\text { DHA } \\
\left.\text { (mg serving }^{-1}\right)\end{array}$ \\
\hline 1 & 3000 & 540 & 360 & 549 & 303 \\
2 & 3000 & 540 & 360 & 492 & 306 \\
3 & 3000 & 540 & 360 & 489 & 291 \\
4 & 3000 & 540 & 360 & 522 & 348 \\
5 & 3000 & 540 & 360 & 591 & 372 \\
6 & 3000 & 540 & 360 & 486 & 282 \\
7 & 3000 & 540 & 360 & 537 & 300 \\
8 & 3000 & 540 & 360 & 510 & 303 \\
9 & 3000 & 540 & 360 & 540 & 318 \\
10 & 600 & 120 & 300 & 136 & 265 \\
\hline
\end{tabular}

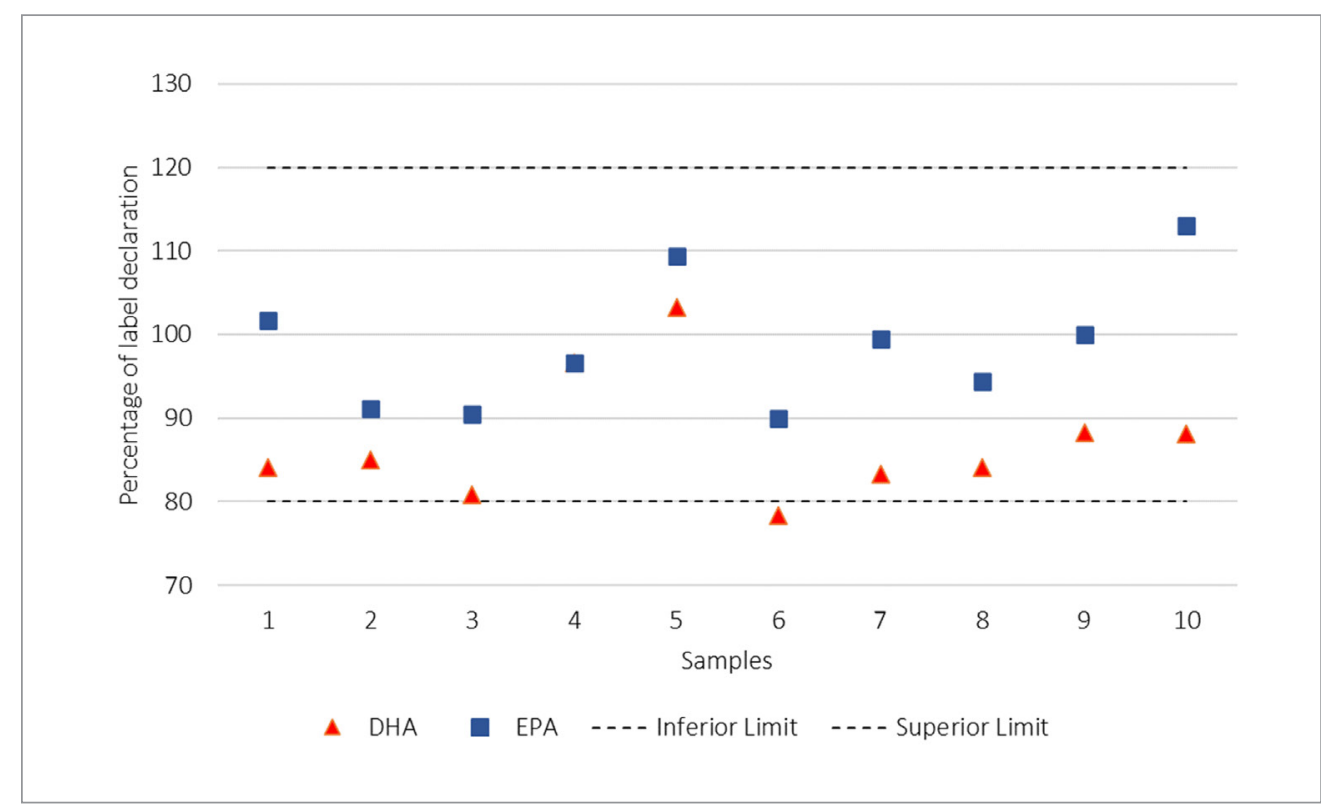

Figure 2. Percentage of eicosapentaenoic $(\square)$ and docosahexaenoic $(\Delta)$ acids content on the label declaration for all samples analyzed. Dotted lines express legal tolerance limits. 
Against the evidences reported by Galuch et al. (2018) [8] that some FO supplements acquired in the Brazilian market (samples acquired in Maringá City, Paraná State) were not a source of EPA and DHA and were adulterated with soybean oil, most FO analyzed within this data set (acquired in Juiz de Fora city, Minas Gerais State), have shown to meet their label claims for EPA and DHA contents, considering the Brazilian current legal tolerance limits. These findings are also contrary to the evidences shown by several international studies, which demonstrated the lack of rigor in the labeling of FO dietary supplements. In the United States, Srigley \& Rader (2015) [7] detected that almost $20 \%$ of the marine oil supplements did not meet their respective label declarations and Kleiner et al. (2015) [15] verified that among all samples considered, over $70 \%$ of the supplements tested did not contain the stated labeled amount of EPA or DHA. In New Zealand, it was observed that only $9 \%$ of the FO supplements analyzed contained quantities of EPA and DHA that were equal or higher than their labeled contents [5] and, more recently, it was revealed that $9 \%$ of the FO samples did not comply with the declarations [16]. In South Africa, only $52 \%$ and $35 \%$ of the FO supplements contained the labeled amount of EPA and DHA, respectively [17], while, in Austria, more than $10 \%$ of the omega-3 supplements did not meet omega-3 labeling [18]. A further study from Australia reported misdeclaration of $42 \%$ for EPA and $10 \%$ for DHA [19], and, in Korea, $100 \%$ of the FO samples were mislabelled according to analytically determined contents [6]. Overall, these findings highlight the quality of FO Brazilian supplements, but also warn the real need to intensify the inspection of dietary FO supplements worldwide in order to guarantee the rights and interests of the consumers.

\section{Evaluation of the delivered fatty acid form in fish oil supplements}

As evidenced in Figure 1, GC-FID was successful in the discrimination between ME and EE because their retention times were slightly different. Omega-3 ME eluted just before the EE homologs (Figure 1C). However, these assignments were also corroborated by GC-MS (Mass spectra of EPA and DHA TAG and EE are available in the supporting information), which permitted to distinguish FA forms in the different supplements due to a $\mathrm{CH}_{2}$ group missing in the ME form $(\mathrm{m} / \mathrm{z}=14)$. EPA ME presented $\mathrm{M}+$ at $m / z=316$, while the EPA EE homolog showed $\mathrm{M}+$ at $m / z=330$. Correspondingly, DHA ME presented $\mathrm{M}+$ at $m / z=$ 342 , and DHA EE exhibited $M+$ at $m / z=356$. Thus, both techniques can be employed to TAG and EE discrimination in FO supplements, under the analytical conditions described.

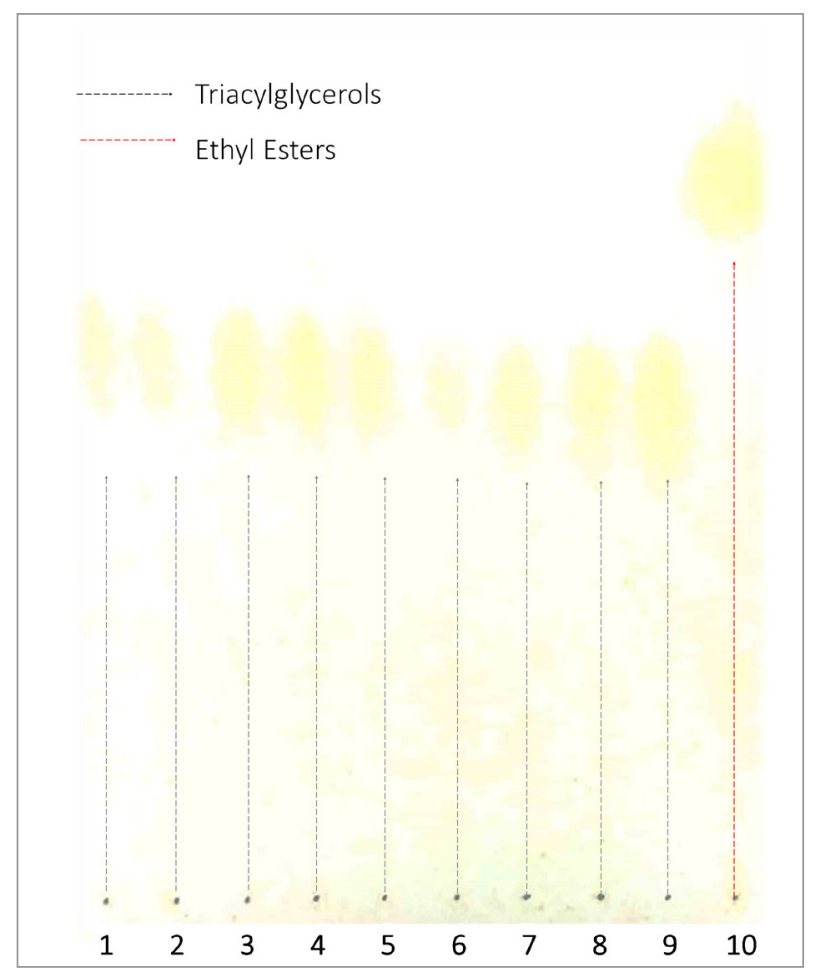

FA form could also be evaluated by TLC, as shown in Figure 3. This method distinguished TAG from EE samples based on the difference in their retention factors. TAG samples resulted in a retention factor $=$ 0.6 and EE samples resulted in a retention factor $=$ 0.8 . However, it is worth noticing that Sample 10 was not observed as a mixture of TAG and EE, as evidenced by GC. Only a spot corresponding to the EE form was visualized. Then, in order to obtain a complete profile of TAG and EE forms in FO samples and also to quantify these forms in supplements, GC techniques are recommended.

Figure 3. Thin-layer chromatography analysis of fish oil supplements containing fatty acids as triacylglycerols (retention factor $=0.6)$ and ethyl esters (retention factor $=$ $0.8)$. 


\section{CONCLUSIONS}

A set of chromatographic techniques (TLC, GC-FID, and GC-MS) was approached in detail in this study applied to FO analyses, considering both FA contents and form. The profiles of natural and concentrated FO with FA present in the form of TAG and EE were compared and discussed, and identified chromatograms were shown, as well as the mass spectra of EPA and DHA methyl and ethyl esters. This information can be useful to new researches, considering FO analyses and also future investigations about the bioavailability of FA forms, which needs to be further approached in the literature. It was possible to differentiate TAG and EE FA by GC-FID, due to the difference in the retention times of both forms, and also by GC-MS, as a result of the different $\mathrm{m} / \mathrm{z}$ spectra observed. TLC was also able to distinguish FA forms, but a mixture of TAG and EE in FO concentrates was not observed, as evidenced by GC techniques. Besides, label accuracy of Brazilian supplements was also evaluated, which could be of interest in a globalized market. All FO analyzed were a source of EPA and DHA, containing from $90 \%$ to $113 \%$ EPA and from $78 \%$ to $103 \%$ DHA, according to the omega- 3 contents declared by manufacturers.

\section{Acknowledgments}

This study was financed in part by the "Coordenação de Aperfeiçoamento de Pessoal de Nível Superior" (CAPES), Brazil - Finance Code 001 and PDSE 88881187078/2018-01; Spanish Ministry of Economy and Competitiveness (AGL2016-75159-C2-2-R); "Conselho Nacional de Desenvolvimento Científico e Tecnológico" (CNPq), (303355/2017-4, 424032/2018-0); "Financiadora de Estudos e Projetos" (Finep), (CT-INFRA 01/2013-REF 0633/13); "Rede Mineira de Quimica" (RQ-MG), (CEX.RED- 00010-14), and "Instituto Nacional de Ciência e Tecnologia de Bioanalítica" (INCTBio), (FAPESP 2014/50867-3, CNPq 465389/2014-7).

\section{Conflicts of interest}

All the authors declare no conflict of interest.

\section{REFERENCES}

1. Gammone, M. A.; Riccioni, G.; Parrinello, G.; D’Orazio, N. Nutrients, 2019, 11, pp 1-16 (https://doi. org/10.3390/nu11010046).

2. Derbyshire, E. Nutrients, 2018, 10, pp 1-18 (https://doi.org/10.3390/nu10081094).

3. Rubio-Rodríguez, N.; Beltrán, S.; Jaime, I.; de Diego, S. M.; Sanz, M. T.; Carballido, J. R. Innov. Food Sci. Emerg. Technol., 2010, 11, pp 1-12 (https://doi.org/10.1016/j.ifset.2009.10.006).

4. Ghasemifard, S.; Turchini, G. M.; Sinclair, A. J. Prog. Lipid Res., 2014, 56, pp 92-108 (https://doi. org/10.1016/j. plipres.2014.09.001).

5. Albert, B. B., Derraik, G. B.; Cameron-Smith, D.; Hofman, P. L.; Tumanov, S.; Albert, B. B.; Villas-Boas, S. G.; Garg, M. L.; Cutfield, W. S. Sci. Rep., 2015, 5, pp 1-7 (https://doi.org/10.1038/srep07928).

6. Lee, J.; Kim, M. K.; Kim, B.; Kim, J.; Lee, K. Int. J. Food Sci. Technol., 2016, 51, pp 2217-2224 (https:// doi.org/10.1111/ijfs.13198).

7. Srigley, C. T.; Rader, J. I. J. Agric. Food Chem., 2015, 62, pp 7268-7278 (https://doi.org/10.1021/ jf5016973).

8. Galuch, M. B.; Carbonera, F.; Magon, T. F. S.; Pizzo, J. S.; Santos, O. O. J. Brazilian Chem. Soc., 2018, 29, pp 631-638 (https://doi.org/10.21577/0103-5053.20170177).

9. Gallardo, B.; Gómez-Cortés, P.; Mantecón, A. R.; Juárez, M.; Manso, T.; de la Fuente, M. A. Animal, 2014, 8, pp 1178-1190 (https://doi.org/10.1017/s1751731114000238).

10. Srigley, C. T.; Orr-Tokle, I. C. Lipids, 2018, 53, pp 749-754 (https://doi.org/10.1002/lipd.12078).

11. Li, Z.; Kotoski, S. P.; Srigley, C. T. J. Am. Oil Chem. Soc., 2019 (https://doi.org/10.1002/aocs.12194).

12. Karunathilaka, S. R.; Hwan, S.; Mossoba, M. M.; Jean, B.; Brückner, L.; Ellsworth, Z.; Srigley, C. T. J. Food Compos. Anal., 2019, 77, pp 9-19 (https://doi.org/10.1016/j.jfca.2018.12.009).

13. Nollet, L. M. L.; Toldra, F. Handbook of Seafood and Seafood Products Analysis. CRC Press, 2009. 
14. Ministério da Saúde do Brasil. Resolução da Diretoria Colegiada - RDC Nº 360. Diário Oficial da União 2003.

15. Kleiner, A. C.; Cladis, D. P.; Santerre, C. R. J. Sci. Food Agric., 2015, 95, pp 1260-1267 (https://doi. org/10.1002/jsfa.6816).

16. Bannenberg, G.; Mallon, C.; Edwards, H.; Yeadon, D.; Yan, K., Johnson, K., Ismail, A., Sci. Rep., 2017, 7, pp 1-13 (https://doi.org/10.1038/s41598-017-01470-4).

17. Opperman, M.; Benade, S. Cardiovasc. J. Afr., 2013, 24, pp 297-302 (https://doi.org/10.5830/CVJA2013-074).

18. Tatarczyk, T.; Engl, J.; Ciardi, C.; Laimer, M.; Kaser, S.; Salzmann, K.; Lenners, R.; Patsch, J. R.; Ebenbichler, C. F. Wien Klin Wochenschr, 2007, 119, pp 417-422 (https://doi.org/10.1007/s00508-0070820-5).

19. Hamilton, K.; Brooks, P.; Holmes, M.; Cunningham, J.; Russell, F. D. Nutr. Diet., 2010, 67, pp 182-189 (https://doi.org/10.1111/j.1747-0080.2010.01453.x). 


\section{SUPPORTING INFORMATION}

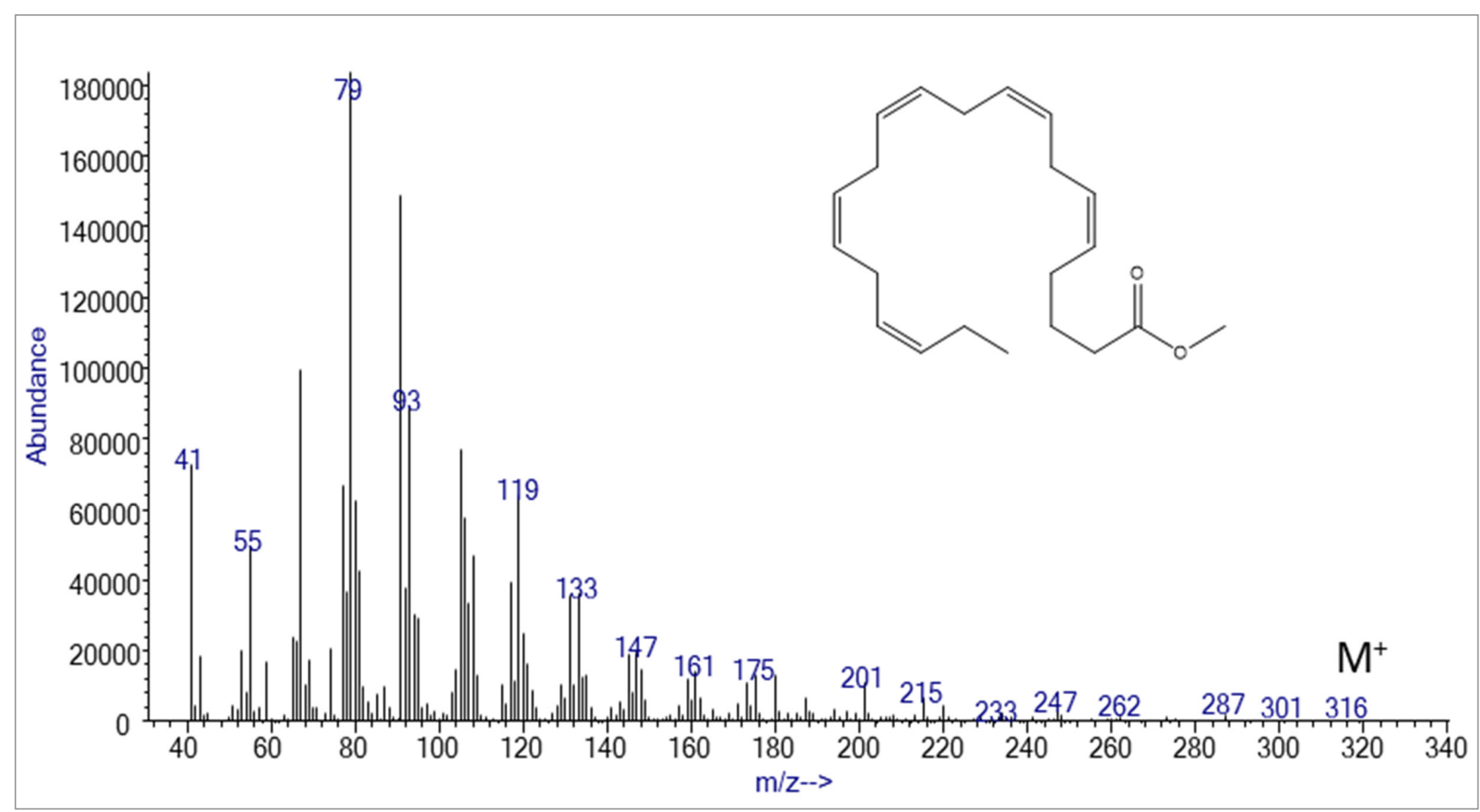

Supporting information - Figure 1. Mass spectra of eicosapentaenoic acid methyl ester.

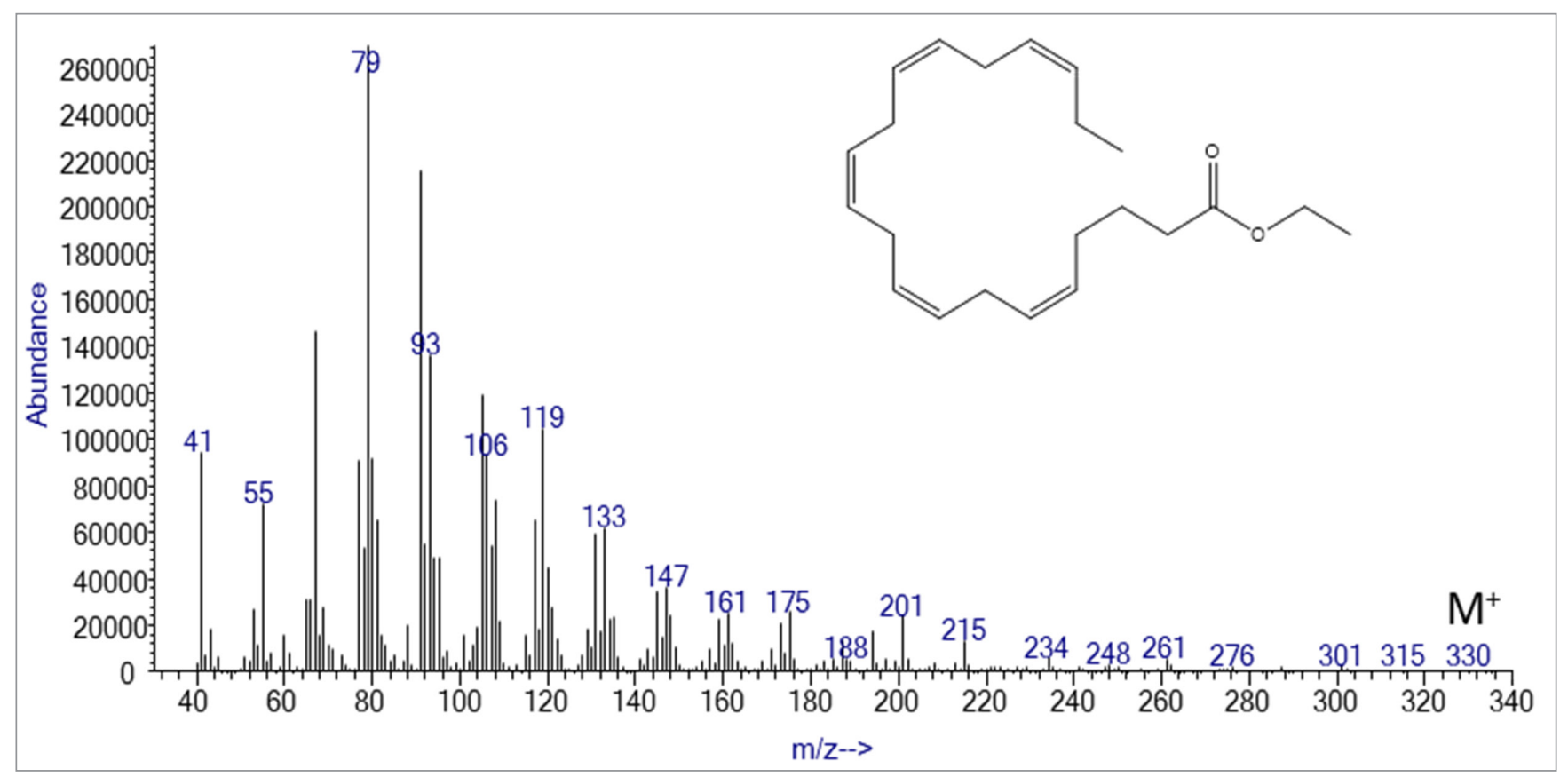

Supporting information - Figure 2. Mass spectra of eicosapentaenoic acid ethyl ester. 


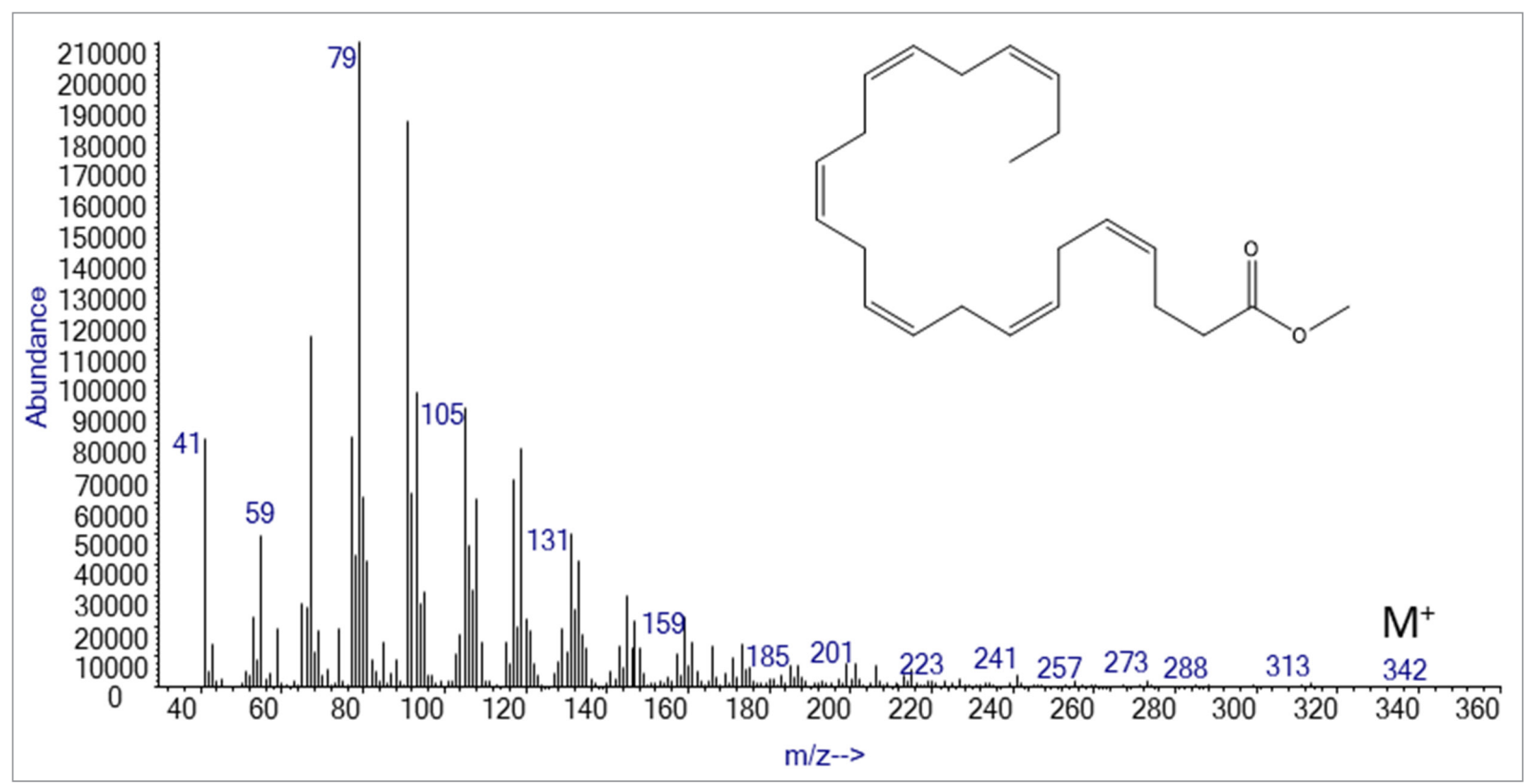

Supporting information - Figure 3. Mass spectra of docosahexaenoic acid methyl ester.

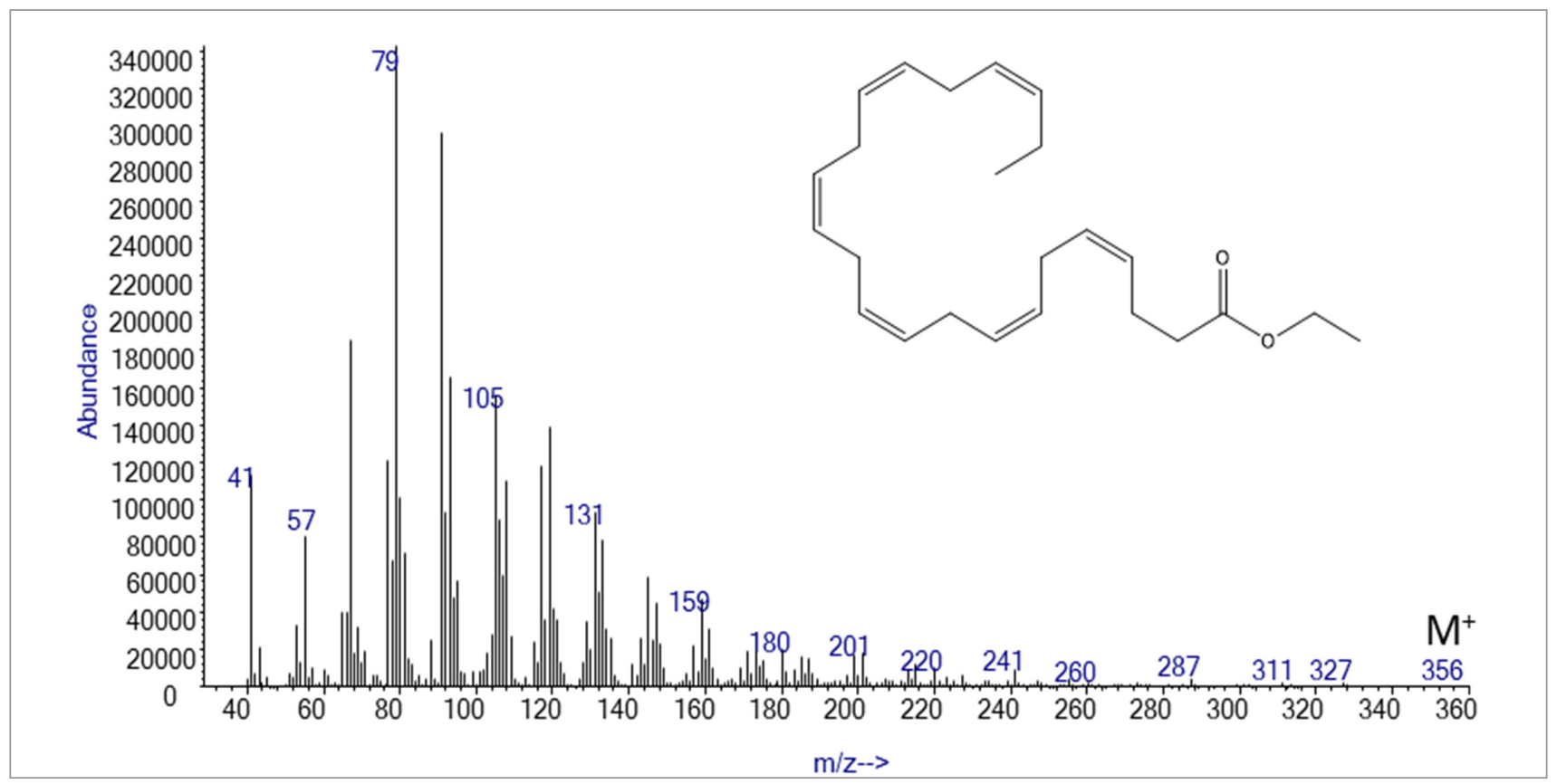

Supporting information - Figure 4. Mass spectra of docosahexaenoic acid ethyl ester. 